\title{
SOME PROPERTIES OF ESSENTIAL SPECTRA OF A POSITIVE OPERATOR
}

\author{
E. A. ALEKHNO (Belarus, Minsk)
}

\begin{abstract}
Let $E$ be a Banach lattice, $T$ be a bounded operator on $E$. The Weyl essential spectrum $\sigma_{\text {ew }}(T)$ of the operator $T$ is a set $\sigma_{\text {ew }}(T)=\underset{K \in \mathcal{K}(E)}{\cap} \sigma(T+K)$, where $\mathcal{K}(E)$ is a set of all compact operators on $E$. In particular for a positive operator $T$ next subsets of the spectrum

$$
\sigma_{\mathrm{ew}}^{+}(T)=\bigcap_{0 \leq K \in \mathcal{K}(E)} \sigma(T+K), \quad \sigma_{\mathrm{ew}}^{-}(T)=\bigcap_{0 \leq K \in \mathcal{K}(E) \leq T} \sigma(T-K)
$$

are introduced in the article. The conditions by which $r(T) \notin \sigma_{\text {ef }}(T)$ implies either $r(T) \notin \sigma_{\text {ew }}^{+}(T)$ or $r(T) \notin \sigma_{\text {ew }}^{-}(T)$ are investigated, where $\sigma_{\text {ef }}(T)$ is the Fredholm essential spectrum. By this reason, the relations between coefficients of the main part of the Laurent series of the resolvent $R(., T)$ of a positive operator $T$ around of the point $\lambda=r(T)$ are studied. The example of a positive integral operator $T: L_{1} \rightarrow L_{\infty}$ which doesn't dominate a non-zero compact operator, is adduced. Applications of results which are obtained, to the spectral theory of band irreducible operators, are given. Namely, the criteria when the operator inequalities $0 \leq S<T$ imply the spectral radius inequality $r(S)<r(T)$, are established, where $T$ is a band irreducible abstract integral operator.
\end{abstract}

Key words: Banach lattice, positive operator, perturbation theory, essential spectrum, irreducible operator, integral operator

MSC 2000: Primary 46B42, 47B65, 47A55, 47A53, 47A10, 47A11; Secondary 47G10

\section{Introduction}

The perturbation theory studies the behaviour of the spectrum of a linear operator $T$ under the perturbation of $T$ by "small", as a rule compact or like to it, operators. This theory was strongly developed in many directions and plural applications were found to wide classes of linear operators on Banach spaces (see, for example, [5, 4]). The notion of an essential spectrum is important in the perturbation theory. So, essential spectra are subsets of the spectrum which are invariant under a perturbation of the given operator by operators of the concrete form. These spectra are obtained, for example, at the expense of the strengthening of the non-invertibility's definition.

On the other hand, the spectral theory of positive operators occupies a major place in the general concept of operators on Banach lattices (see, for example, monographs $[1,10]$ which 
can acquaint with main stages of the development and the achievements in this direction). However, in spite of numerous attempts the general operator theory is united and connected with the theory of positive operators, the next question have receive almost no attentions. How can results of the perturbation theory be define more precisely and what form they receive in the case of positive operators on Banach lattices? To make some contribution in this direction is main purpose of this note.

Let $E$ be an infinite dimensional Banach lattice, $T$ be a linear bounded operator on $E$. As usual, the spectrum of an operator $T$ will be denoted by $\sigma(T)$. Recall that the Fredholm essential spectrum of an operator $T$ is called the set

$$
\sigma_{\text {ef }}(T)=\{\lambda \in \mathbb{C}: \lambda-T \text { is not a Fredholm operator }\}
$$

and the Weyl essential spectrum is called the set $\sigma_{\text {ew }}(T)=\bigcap_{K \in \mathcal{K}(E)} \sigma(T+K)$, where $\mathcal{K}(E)$ is the set of all compact operators on $E$. For a positive operator $T$ next subsets of the spectrum are also introduced:

$$
\sigma_{\text {ew }}^{+}(T)=\bigcap_{0 \leq K \in \mathcal{K}(E)} \sigma(T+K), \quad \sigma_{\text {ew }}^{-}(T)=\bigcap_{0 \leq K \in \mathcal{K}(E) \leq T} \sigma(T-K) .
$$

It is clear that inclusions

$$
\sigma_{\text {ef }}(T) \subseteq \sigma_{\text {ew }}(T) \subseteq \sigma_{\text {ew }}^{-}(T) \cap \sigma_{\text {ew }}^{+}(T) \subseteq \sigma_{\text {ew }}^{-}(T) \cup \sigma_{\text {ew }}^{+}(T) \subseteq \sigma(T)
$$

hold. There exist wide classes of operators such that rarely the equality $\sigma_{\text {ef }}(T)=\sigma(T)$ holds. For example, if $T$ is a strictly singular operator on $E$, then ([1], p. 314) $\sigma_{\text {ef }}(T)$ is equal $\{0\}$, thus, generally speaking, the inclusion $\sigma_{\text {ef }}(T) \subseteq \sigma(T)$ is strict. The forward shift operator $T$ on the space $\ell_{p}(1<p<\infty), T\left(x_{1}, x_{2}, \ldots\right)=\left(0, x_{1}, \ldots\right)$, gives the example of a positive operator such that ([4], p. 72-73)

$$
\sigma_{\mathrm{ef}}(T)=\{\lambda:|\lambda|=1\} \subset \sigma_{\mathrm{ew}}(T)=\{\lambda:|\lambda| \leq 1\} .
$$

But among of points of the spectrum of a positive operator there exists one "special", namely $\lambda=r(T)$, it is a point corresponding to the spectral radius of a positive operator $T$ (recall that ([1], p. 276) for a positive operator $T$ on a Banach lattice $E$ the inclusion $r(T) \in \sigma(T)$ always holds). For example, in many cases by singularities of the spectrum at a neighbourhood of this point, it can judge about properties of all spectrum or at least of the peripheral spectrum of a positive operator.

It will be show below that under the number of the additional assumptions the condition $r(T) \notin \sigma_{\text {ef }}(T)$ implies $r(T) \notin \sigma_{\text {ew }}^{-}(T) \cup \sigma_{\text {ew }}^{+}(T)$ for a positive operator $T$. The last part of the note is devoted to the application of obtained results to the question whether the operator inequalities $0 \leq S<T$ imply the spectral radius inequality $r(S)<r(T)$, where $T$ is a band irreducible abstract integral operator.

For terminology, notions, and properties on the theory of Banach lattices and positive operators not explained or proved in this note, we refer to [1,3]; see also [10]. Throughout the note, unless otherwise stated, a Banach lattice $E$ will be assumed to be infinite dimensional and an operator $T$ will be assumed positive. 


\section{When does $r(T) \notin \sigma_{\text {ef }}(T)$ imply $r(T) \notin \sigma_{\text {ew }}^{+}(T)$ ?}

Let $r(T) \notin \sigma_{\text {ef }}(T)$. It is equivalent that ([1], p. 300-302) $r(T)>0$, the point $\lambda=r(T)$ is a pole of the resolvent $R(., T)$ of the operator $T$ and the residuum $T_{-1}$ of the function $R(., T)$ at this point presents the operator of a finite-rank. It is no loss of generality to assume that $r(T)=1$. Consider the Laurent series expansion of the resolvent function $R(., T)$ around $\lambda=1$

$$
R(\lambda, T)=\frac{1}{(\lambda-1)^{m}} T_{-m}+\ldots+\frac{1}{\lambda-1} T_{-1}+T_{0}+(\lambda-1) T_{1}+\ldots,
$$

where $m \geq 1$ is the order of the pole of $R(., T)$ at $\lambda=1$. Operators $T_{i}\left(T_{i}=0\right.$, when $i<-m)$ are defined on the complexification $E_{\mathbb{C}}$ of the Banach lattice $E$ and are real maps. The restriction of $T_{i}$ to $E$ will denote by $T_{i}$ again. In particular the operator $T_{-m} \geq 0$ and the relations $T_{-i} T_{-j}=T_{-i-j+1}, i, j \geq 1$ and $T_{i} T_{j}=0, i \geq 0, j \leq-1$ hold. Note also that all operators $T_{-i}, i \geq 1$ are of a finite-rank, therefore ([3], p. 272) modules of them exist and are compact operators, moreover ([10], p. 296) $\left|T_{-i}\right|^{*}=\left|T_{-i}^{*}\right|$. In next lemmas the number of necessary in the future properties of operators $T_{i}$ are adduced.

Lemma 1. Under the above assumptions the next equalities

$$
T_{-m}\left|T_{-1}\right|=\left|T_{-1}\right| T_{-m}=T_{-m}, \quad\left|T_{-i}\right| T_{-m}=T_{-m}\left|T_{-i}\right|=0
$$

hold for every $i>1$.

Proof. In cases $m=1$ and $i>m \geq 1$ the assertions of lemma are obvious.

Let the Banach lattice $E$ be Dedekind complete and the operator $T_{-m}$ be order continuous. Fix an arbitrary element $x \geq 0$. Then, with the help of the equality $T_{-m} R(\lambda, T)=\frac{1}{\lambda-1} T_{-m}$, we have for $m \geq 2$

$$
\begin{gathered}
T_{-m}\left|T_{-(m-1)} x\right|=\lim _{\lambda \downarrow 1}(\lambda-1)^{m-1} T_{-m}\left|R(\lambda, T) x-\frac{1}{(\lambda-1)^{m}} T_{-m} x\right|= \\
=\lim _{\lambda \downarrow 1}(\lambda-1)^{m-1} T_{-m}\left|R(\lambda, T) x-\frac{1}{(\lambda-1)^{m-1}} T_{-m} R(\lambda, T) x\right| \leq \\
\leq \lim _{\lambda \downarrow 1}(\lambda-1)^{m-1} T_{-m} R(\lambda, T)\left|x-\frac{1}{(\lambda-1)^{m-1}} T_{-m} x\right| \leq \lim _{\lambda \downarrow 1}(\lambda-1)^{m-2} T_{-m} x .
\end{gathered}
$$

Therefore by $m>2 T_{-m}\left|T_{-(m-1)} x\right|=0$ and by $m=2 T_{-2}\left|T_{-1} x\right| \leq T_{-2} x$. Using the relation ([3], p. 15, theorem 1.16)

$$
\left\{\sum_{i=1}^{n}\left|T_{-(m-1)} x_{i}\right|: \sum_{i=1}^{n} x_{i}=x, x_{i} \geq 0\right\} \uparrow\left|T_{-(m-1)}\right| x,
$$

we have for $m>2 T_{-m}\left|T_{-(m-1)}\right|=0$ and for $m=2 T_{-2}\left|T_{-1}\right| \leq T_{-2}$. With the help of the elementary induction and the equality $(k \geq 1)$

$$
T_{-(m-k)}=\lim _{\lambda \downarrow 1}(\lambda-1)^{m-k}\left(R(\lambda, T)-\frac{1}{(\lambda-1)^{m}} T_{-m}-\ldots-\frac{1}{(\lambda-1)^{m-k+1}} T_{-(m-k+1)}\right)
$$


we prove analogously that for $m>k+1 T_{-m}\left|T_{-(m-k)}\right|=0$, for $m=k+1 T_{-m}\left|T_{-1}\right| \leq T_{-m}$. On the other hand, obviously $T_{-m}=\left|T_{-m} T_{-1}\right| \leq T_{-m}\left|T_{-1}\right|$ thus $T_{-m}=T_{-m}\left|T_{-1}\right|$ for all $m>2$.

In the general case, the equalities

$$
T_{-m}^{*}=T_{-m}^{*}\left|T_{-1}^{*}\right|=\left(\left|T_{-1}\right| T_{-m}\right)^{*}, T_{-m}^{* *}=T_{-m}^{* *}\left|T_{-1}^{* *}\right|=\left(T_{-m}\left|T_{-1}\right|\right)^{* *}
$$

hold as Banach lattices $E^{*}$ and $E^{* *}$ are Dedekind complete and operators $T_{-m}^{*}$ and $T_{-m}^{* *}$ are order continuous, hence $T_{-m}=\left|T_{-1}\right| T_{-m}=T_{-m}\left|T_{-1}\right|$. The proof of the remain part of the lemma is entirely analogous.

With the help of the matrix

$$
\left(\begin{array}{cccc}
\frac{1}{2} & 0 & 0 & 0 \\
a_{1} & 1 & 0 & 0 \\
a_{2} & a_{3} & 1 & 0 \\
a_{4} & a_{5} & a_{6} & \frac{1}{2}
\end{array}\right)
$$

we can show that, generally speaking, the next equalities

$$
\left|T_{-1}\right|^{2}=\left|T_{-1}\right|, \quad\left|T_{-1}\right| T_{-1}=T_{-1}, \quad T_{-1}\left|T_{-1}\right|=\left|T_{-1}\right|
$$

don't hold.

Lemma 2. For arbitrary real numbers $\beta_{1}, \ldots, \beta_{m-1}$ the equality

$$
T_{-m}\left(T_{-1}+\beta_{1} T_{-2}+\ldots+\beta_{m-1} T_{-m}\right)^{+}=T_{-m}
$$

holds.

Proof. The desired assertion follows from lemma 1 and the next chain of the relations

$$
\begin{gathered}
T_{-m}=T_{-m}\left(T_{-1}+\beta_{1} T_{-2}+\ldots+\beta_{m-1} T_{-m}\right) \leq T_{-m}\left(T_{-1}+\beta_{1} T_{-2}+\ldots+\beta_{m-1} T_{-m}\right)^{+} \leq \\
\leq T_{-m}\left|T_{-1}+\beta_{1} T_{-2}+\ldots+\beta_{m-1} T_{-m}\right| \leq T_{-m}\left|T_{-1}\right|=T_{-m} .
\end{gathered}
$$

Lemma 3. Under the assumptions that $E$ is either $A M$ - or $A L$-space and $m>1$, there exist sequences $a_{n}^{(1)}, \ldots, a_{n}^{(m-1)}$ that converge to $+\infty$ and

$$
\lim _{n \rightarrow \infty}\left(T_{-1}+a_{n}^{(1)} T_{-2}+\ldots+a_{n}^{(m-1)} T_{-m}\right)^{-}=0 .
$$

Proof. We can assume that $E$ is $A L$-space. Using the elementary induction we show more: for each $k, 1 \leq k<m$ there exist sequences $a_{n}^{(1)}, \ldots, a_{n}^{(m-k)}$ that converge to $+\infty$ and

$$
\lim _{n \rightarrow \infty}\left(T_{-k}+a_{n}^{(1)} T_{-(k+1)}+\ldots+a_{n}^{(m-k)} T_{-m}\right)^{-}=0 .
$$

Hence by $k=1$ we obtain the desired assertion /!/. First of all we show that the assertion /!!/ is true for $k=m-1$. Fix a natural $n$. Find $\lambda_{n}, 1+\frac{1}{n} \geq \lambda_{n}>1$ such that

$$
\left\|T_{-(m-1)}-\left(\left(\lambda_{n}-1\right)^{m-1} R\left(\lambda_{n}, T\right)-\frac{1}{\lambda_{n}-1} T_{-m}\right)\right\| \leq \frac{1}{n} .
$$


Then put $a_{n}=\frac{1}{\lambda_{n}-1}$ and with the help of the relations $R\left(\lambda_{n}, T\right) \geq 0$ and $\|S\|=\||S|\|$ for every $S \in \mathcal{L}(E)$ we have $\left\|\left(T_{-(m-1)}+a_{n} T_{-m}\right)^{-}\right\| \leq \frac{1}{n}$. Let us assume that /!!/ is proved for $k+1, \ldots, m-1$, where $1 \leq k<m-1$. We show that it is true for $k$. For $n \in \mathbb{N}$ find $\lambda_{n}$, $1+\frac{1}{n} \geq \lambda_{n}>1$ such that

$$
\left\|T_{-k}-\left(\left(\lambda_{n}-1\right)^{k} R\left(\lambda_{n}, T\right)-\frac{1}{\left(\lambda_{n}-1\right)^{m-k}} T_{-m}-\ldots-\frac{1}{\lambda_{n}-1} T_{-(k+1)}\right)\right\| \leq \frac{1}{2 n} .
$$

There exist numbers $a_{1}, \ldots, a_{m-(k+1)} \geq 0$ for which the inequality

$$
\left\|\left(T_{-(k+1)}+a_{1} T_{-(k+2)}+\ldots+a_{m-(k+1)} T_{-m}\right)^{-}\right\| \leq \frac{1}{2 n}
$$

holds. Then put

$$
a_{n}^{(1)}=\frac{1}{\lambda_{n}-1}+1, \quad a_{n}^{(i)}=a_{i-1}+\frac{1}{\left(\lambda_{n}-1\right)^{i}}, \quad 1<i \leq m-k
$$

we obtain

$$
\left\|\left(T_{-k}+a_{n}^{(1)} T_{-(k+1)}+\ldots+a_{n}^{(m-k)} T_{-m}\right)^{-}\right\| \leq \frac{1}{n}
$$

and the proof is finished.

From lemma 3 follows that in the case $m=2 \lim _{n \rightarrow \infty}\left(T_{-1}+n T_{-2}\right)^{-}=0$. When a Banach lattice $E$ is a finite dimensional it can assert more, namely by sufficiently large $n$ it is the positivity of matrices $T_{-1}+n T_{-2}$.

As far as the author knows, lemmas 1 - 3 seems to be new even for case of matrices. Note also that the assertions in these lemmas have an algebraic form, therefore it must stay true for elements of an arbitrary Banach algebra which is equipped with a partial order by a positive cone.

Theorem 4. Let $T$ be a positive operator on a Banach lattice $E$ such that $r(T) \notin \sigma_{\mathrm{ef}}(T)$. Then each of the following conditions ensures that $r(T) \notin \sigma_{\mathrm{ew}}^{+}(T)$ :

(a) the residuum $T_{-1}$ of the resolvent $R(., T)$ at the point $\lambda=r(T)$ is a positive operator. In particular it is true in the case, when $\lambda=r(T)$ is a simply pole of $R(., T)$;

(b) $r(T)$ is a simply eigenvalue of the operator $T$, i.e. $\operatorname{dim} N(r(T)-T)=1$;

(c) the Banach lattice $E$ is either AM- or AL-space and $\lambda=r(T)$ is a pole of $R(., T)$ of an order two.

Proof. Suppose that $r(T)=1$.

(a) It is enough to prove that $1 \notin \sigma\left(T+T_{-1}\right)$. Assume by the way of a contradiction. Then the point $\lambda=1$ is a pole of a finite-rank of the resolvent $R\left(., T+T_{-1}\right)$ of the operator $T+T_{-1}$ as $1 \notin \sigma_{\text {ef }}\left(T+T_{-1}\right)$. Therefore there exists a non-zero $x$ for which $T x+T_{-1} x=x$, hence $T_{-m} x=T_{-m}\left(T x+T_{-1} x\right)=2 T_{-m} x$, so $T_{-m} x=0$. By the induction it is easily to show that $T_{-(m-1)} x=\ldots=T_{-1} x=0$ thus $T x=x$ and the equalities $x=T_{-1} x=0$ follow. We get a contradiction.

(b) There exist a functional $z^{*} \geq 0$ and an element $z \geq 0$ such that $z^{*}\left(T_{-m} z\right)>0$. Show that $1 \notin \sigma\left(T+z^{*} \otimes z\right)$. Assume by the way of a contradiction, choose a non-zero $x$ for which $x=T x+\left(z^{*} x\right) z$. This equality implies $\left(z^{*} x\right) T_{-m} z=0$ whence $z^{*} x=0$, so $x=T x$. 
On the other hand, $T_{-m} z \in N(T-I)$ or $T_{-m} z=\beta x$ for some $\beta$. As a result, we have $z^{*}\left(T_{-m} z\right)=\beta z^{*} x=0$, it contradicts to the choice of $z^{*}$ and $z$.

(c) If $1 \in \sigma_{\mathrm{ew}}^{+}(T)$ then for every $n$ there exists $x_{n},\left\|x_{n}\right\|=1$ such that $x_{n}=T x_{n}+$ $\left(T_{-1}+n T_{-2}\right)^{+} x_{n}$. Use lemmas 2 and 3 we have $T_{-2} x_{n}=0$ and $x_{n}-T x_{n}-T_{-1} x_{n} \rightarrow 0$ whence

$$
T_{-1} x_{n}=T_{-1}\left(T_{-1} x_{n}+T x_{n}-x_{n}\right) \rightarrow 0,
$$

so

$$
x_{n}-T x_{n} \rightarrow 0 \text {. }
$$

The operator $T-I$ is a Fredholm operator, therefore ([4], p. 56; see also [5], p. 233) the sequence $x_{n}$ has a convergent subsequence. It is no loss of generality to assume that $x_{n} \rightarrow x_{0}$, $\left\|x_{0}\right\|=1$. Then /!/ and /!!/ imply, respectively, $T_{-1} x_{0}=0$ and $T x_{0}=x_{0}$, that gives a contradiction.

The condition of the part (b) is equivalent to the condition that $m$ is equal to the algebraic multiplicity $k$ of the eigenvalue $r(T)$ of $T$ (recall that $k=\operatorname{dim} R\left(T_{-1}\right)=\operatorname{dim} N\left((r(T)-T)^{m}\right)$ ). As showed in the proof of theorem 4, in this case we can perturb the operator $T$ by a rankone positive operator $K$ such that $r(T) \notin \sigma(T+K)$ (note that this follows easily also from the first W-A formula ([5], p. 244-250)). By this reason we note that if there exists an operator $K$ of a rank not greater $t$ (not necessary positive) for which $r(T) \notin \sigma(T+K)$ then $\operatorname{dim} N(r(T)-T) \leq t$. If $R\left(T_{-m}\right)=N(r(T)-T)$ then the adverse is true. It follows that we can perturb $T$ by an operator $K$ of the rank-one such that $r(T) \notin \sigma(T+K)$ iff $\operatorname{dim} N(r(T)-T)=1$.

Note also that lemma 3 is true for the case of an arbitrary Dedekind complete Banach lattice $E$ such that for every order bounded operator $S\|S\|=\||S|\|$. It follows that in the part (c) of theorem 4 sufficiently only this property is assumed. Moreover it is clear that theorem 4 is true when $E$ is a finite dimensional space. It is not known if theorem 4 is valid for an arbitrary Banach lattice $E$.

\section{$3 \quad$ When does $r(T) \notin \sigma_{\text {ef }}(T)$ imply $r(T) \notin \sigma_{\text {ew }}^{-}(T)$ ?}

Theorem 5. Let $T$ be a positive operator on a Banach lattice $E$ such that $r(T) \notin \sigma_{\mathrm{ef}}(T)$ and there exists a net of compact operators $K_{\alpha}$ such that

$$
0 \leq K_{\alpha} x \uparrow T x
$$

for all $x \geq 0$. Then each of the following conditions ensures that $r(T) \notin \sigma_{\mathrm{ew}}^{-}(T)$ :

(a) the point $\lambda=r(T)$ is a simply pole of the resolvent $R(., T)$, moreover the residuum at this point is a strictly positive operator;

(b) E is a Banach lattice with order continuous norm.

Proof. Suppose that $r(T)=1$.

(a) Assume by the way of a contradiction that is $1 \in \sigma_{\text {ew }}^{-}(T)$. Then for all $\alpha$ the equalities $r\left(T-K_{\alpha}\right)=1$ hold, therefore there exist elements $x_{\alpha}>0$ such that $T x_{\alpha}-K_{\alpha} x_{\alpha}=x_{\alpha}$. We have $T_{-1} x_{\alpha}-T_{-1} K_{\alpha} x_{\alpha}=T_{-1} x_{\alpha}$ whence

$$
K_{\alpha} x_{\alpha}=0
$$


so $T x_{\alpha}=x_{\alpha}$. Thus $T_{-1} x_{\alpha}=x_{\alpha}$ or

$$
x_{\alpha} \in T_{-1}(E) .
$$

The projection $T_{-1}$ is a strictly positive, it follows that $T_{-1}(E)$ is a sublattice of $E$ ([1], p. 216). Let $e_{1}, \ldots, e_{l} \geq 0$ be a basis of $T_{-1}(E)$ such that $\left(T_{-1}(E)\right)^{+}=\left\{\sum_{i=1}^{l} \beta_{i} e_{i}: \beta_{i} \geq 0\right\}$ (such basis exists; in fact ([10], p. 70), $T_{-1}(E)$ is order isomorphic $\mathbb{R}^{l}$, where $l=\operatorname{dim} T_{-1}(E)$ ). Then $K_{\alpha} e_{i} \uparrow T e_{i}=e_{i}$ for all $i=1, \ldots, l$, so for some $\alpha_{0}$ operators $K_{\alpha}, \alpha \geq \alpha_{0}$, are strictly positive on the lattice $T_{-1}(E)$. As a result, from /!/ and /!!/ we conclude $x_{\alpha}=0$ by $\alpha \geq \alpha_{0}$, which is a contradiction.

(b) The main idea of the underlying arguments is borrowed from the proof of Lotz-Schaefer theorem ([10], p. 331-332, theorem 5.5). For the proof we use induction on an order $m$ of a pole of the resolvent $R(., T)$ at the point $\lambda=1$. Let $m=1$. Through $J$ is denoted a null ideal of the operator $T_{-1}, J=\left\{x: T_{-1}|x|=0\right\}$. Introduce the operator $T_{J}$ on the Banach lattice $E / J$, $T_{J}(x+J)=T x+J$. Then the operator $T_{J}$ is well defined and satisfies all conditions of the part (a), that is positive, $r\left(T_{J}\right)=1$, the point $\lambda=1$ is a simply pole of the resolvent $R\left(., T_{J}\right)$, the residuum at this point is a strictly positive, moreover the equality $\left(T_{J}\right)_{-1}=\left(T_{-1}\right)_{J}$ holds. We explain the latter statement. Indeed, if $x+J \geq 0$ then

$$
\begin{gathered}
\left(T_{J}\right)_{-1}(x+J)=\lim _{\lambda \downarrow 1}(\lambda-1) R\left(\lambda, T_{J}\right)(x+J)= \\
=\lim _{\lambda \downarrow 1}(\lambda-1)(R(\lambda, T) x+J)=\lim _{\lambda \downarrow 1}(\lambda-1) R(\lambda, T) x+J=T_{-1} x+J>0
\end{gathered}
$$

(if $T_{-1} x+J=0$ then for some $z \in J \quad 0 \leq T_{-1}(x+z) \in J$ and $x+z \geq 0$, it follows that $0=T_{-1}\left|T_{-1}(x+z)\right|=T_{-1}(x+z)$ whence $x+z \in J$ or $\left.x \in J\right)$.

The relations $T_{-1}\left|K_{\alpha} x\right| \leq T_{-1} K_{\alpha}|x| \leq T_{-1}|x|$ imply $K_{\alpha}(J) \subseteq J$ for all $\alpha$, so operators $\left(K_{\alpha}\right)_{J}: E / J \rightarrow E / J$ are well defined and are compact. Further, by the order continuity of the norm $K_{\alpha} x \rightarrow T x$ in $E$ hence $K_{\alpha} x+J \rightarrow T x+J$ in $E / J$, it follows that $\left(K_{\alpha}\right)_{J} \uparrow T_{J}$. By the proven above $r\left(T_{J}-\left(K_{\alpha}\right)_{J}\right)<1$ by $\alpha \geq$ some $\alpha_{0}$. On the other hand, the inequalities $r\left(\left.T\right|_{J}-\left.K_{\alpha}\right|_{J}\right) \leq r\left(\left.T\right|_{J}\right)<1$ hold, where $\left.T\right|_{J},\left.K_{\alpha}\right|_{J}$ are restrictions of operators $T$ and $K_{\alpha}$ to the ideal $J$, respectively. Finally, from the inclusion ([10], p. 352)

$$
\sigma\left(T-K_{\alpha}\right) \subseteq \sigma\left(\left(T-K_{\alpha}\right)_{J}\right) \cup \sigma\left(\left.\left(T-K_{\alpha}\right)\right|_{J}\right)
$$

we have $r\left(T-K_{\alpha}\right)<1$ by $\alpha \geq \alpha_{0}$.

Consider the case of a pole of order $m>1$. Put $J=\left\{x: T_{-m}|x|=0\right\}$. Then the function $R\left(.,\left.T\right|_{J}\right)$ has at the point $\lambda=1$ a pole of order $<m$ while on $E / J R\left(., T_{J}\right)$ has at the point $\lambda=1$ of a first order pole. Clearly $\left.\left.K_{\alpha}\right|_{J} \uparrow T\right|_{J}$ and $\left(K_{\alpha}\right)_{J} \uparrow T_{J}$ whence $r\left(T-K_{\alpha}\right)<1$ by $\alpha \geq$ some $\alpha_{0}$.

In the proof of theorem 5 (the part (b)) only an order continuity of the canonical lattice homomorphism $E \rightarrow E / J$ for an arbitrary closed ideal $J$ is used. However, this, in it's turn, implies that ([3], p. 94) every closed ideal is a band, it follows that by Ando theorem ([9], p. $355)$ the Banach lattice $E$ has an order continuous norm. If the condition (A) is replaced by stronger: there exists a sequence of compact operators $K_{n}$ such that

$$
0 \leq K_{n} x \uparrow T x
$$


for all $x \geq 0$ then, as to see from the proof, the assertion of theorem 5 remains true if only the $\sigma$-order continuity of homomorphism $E \rightarrow E / J$ for every closed ideal $J$ is assumed. It means ([3], p. 94) that every closed ideal in $E$ is a $\sigma$-ideal. The latter is equivalent ([9], p. 353-355) to the $\sigma$-order continuity of a norm on $E$. It is not known if theorem 5 is valid for an arbitrary Banach lattice E.

By the reason of the part (a) of theorem 5 note that the next statement is valid: if for the operator $T$ the conditions of the part (a) and $r(T) \notin \sigma_{\mathrm{ef}}(T)$ hold then the operator inequalities $0 \leq S \leq T$, under the condition of the strictly positivity of the operator $S$ on $T_{-1}(E)$, imply $r(T-S)<r(T)$.

Clearly that in the case of a Dedekind complete Banach lattice $E$ the conditions (A) and $\left(\mathrm{A}^{\prime}\right)$ denote the order convergence in the space of order bounded operators $\mathcal{L}_{b}(E)$. In some cases the condition (A) implies the compactness of $T$. For example, it is when the operator $T$ on a Dedekind complete Banach lattice $E$ has order continuous norm that by Dodds-Fremlin theorem is equivalent to $M$ - and $L$-weak compactness of $T$ (see [3], §18). By the reason of example 6 below we note that such operator can't act on non-atomic spaces either $L_{1}$ or $L_{\infty}$.

Not every positive operator $T$ satisfies the condition (A). For example, this condition doesn't hold when [8]:

(i) $E=L_{1}$ on a space with non-atomic measure, $T>0$ is either a lattice homomorphism or an interval preserving operator;

(ii) $E=L_{\infty}$ on a space with non-atomic measure, $T>0$ is an orthomorphism.

In these cases we can assert even more, namely, the next condition doesn't hold for a positive operator $T$ : there exists a compact operator $K$ for which

$$
0<K \leq T
$$

Clearly that for $T>0$ the condition (A) implies (B). Moreover ([3], p. 283) if $E$ is an arbitrary Dedekind complete Banach lattice then for every operator $T>0$ the condition (B) holds iff $E$ is a discrete Banach lattice; in this case also the condition (A) is valid for every $T>0$.

What can say about the validity of the condition (B) in the case when $T$ is a positive integral operator on some Banach function space $X$ ? It is not known if always an integral operator $T: X \rightarrow X, T>0$, dominates some non-zero positive compact operator. It is true, for example, when $X=L_{p}(1<p<\infty)$. For the case of an integral operator $T: L_{1} \rightarrow L_{\infty}$ the answer is negative. Here is a corresponding

Example 6. Consider the segment $[0,1]$ equipped with its Lebesgue measure $\mu$. There exists [11] a measurable set $E \subseteq[0,1] \times[0,1]$ such that for all sets $E_{1}, E_{2} \subseteq[0,1], \mu\left(E_{i}\right)>0$, $i=1,2$, the inequalities

$$
(\mu \times \mu)\left(E \cap\left(E_{1} \times E_{2}\right)\right)>0, \quad(\mu \times \mu)\left(\left(E_{1} \times E_{2}\right) \backslash E\right)>0
$$

hold. Then the positive integral operator $T$ with the kernel $\chi_{E}$,

$$
T x(t)=\int_{0}^{1} \chi_{E}(t, s) x(s) d \mu(s),
$$

acts from the space $L_{1}[0,1]$ into the space $L_{\infty}[0,1]$. Let $K$ be some compact operator for which the inequalities $0 \leq K \leq T$ hold. Then by Bukhvalov-Schep theorem ([1], p. 184) $K$ is an 
integral operator. Denote the kernel of it by $k(t, s)$ and show that $k(t, s)=0$. Assume by the way of a contradiction, choose a measurable set $E_{0} \subseteq E$ such that $(\mu \times \mu)\left(E_{0}\right)>0$ and $k(t, s) \geq \epsilon>0$ on $E_{0}$. By the compactness of $K$ the function $\omega(t)=k(t,),. t \in[0,1]$, with values into the space $L_{\infty}[0,1]$ is a essentially compact ([6], p. 107), that is there exists a measurable set $\Omega_{0} \subseteq[0,1], \mu\left(\Omega_{0}\right)=1$ such that the set of functions $\left\{\omega(t): t \in \Omega_{0}\right\}$ is relatively compact in $L_{\infty}[0,1]$. Next, take a disjoint partition of the segment $[0,1]$ on measurable sets $B_{1}, \ldots, B_{n}$ such that ([6], p. 4)

$$
\operatorname{ess~sup}_{s_{1}, s_{2} \in B_{i}}\left|k\left(t, s_{1}\right)-k\left(t, s_{2}\right)\right|<\epsilon
$$

for all $i=1, \ldots, n$ and every $t \in \Omega_{0}$. For some $i_{0}(\mu \times \mu)\left(\left([0,1] \times B_{i_{0}}\right) \cap E_{0}\right)>0$. The relations

$$
B_{i_{0}} \cap\left(E_{0}\right)_{t}=\left(\left([0,1] \times B_{i_{0}}\right) \cap E_{0}\right)_{t}
$$

and

$$
(\mu \times \mu)\left(\left([0,1] \times B_{i_{0}}\right) \cap E_{0}\right)=\int_{0}^{1} \mu\left(\left(\left([0,1] \times B_{i_{0}}\right) \cap E_{0}\right)_{t}\right) d \mu(t)>0
$$

imply the validity of the inequality $\mu\left(B_{i_{0}} \cap\left(E_{0}\right)_{t}\right)>0$ for all $t$ from some set $A \subseteq[0,1]$ with the positive measure (recall that for $D \subseteq[0,1] \times[0,1] D_{t}=\{s \in[0,1]:(t, s) \in D\}$, where $t \in[0,1])$. Put $C=\left(A \times B_{i_{0}}\right) \backslash E$. Then from /!/ $(\mu \times \mu)(C)>0$ and $\mu\left(C_{t_{*}}\right)>0$ for some $t_{*} \in A \cap \Omega_{0}$. Note that $C_{t_{*}} \subseteq B_{i_{0}}$. Moreover sets $C_{t_{*}}$ and $\left(E_{0}\right)_{t_{*}}$ are disjoint. Actually if $s \in\left(E_{0}\right)_{t_{*}}$ then $\left(t_{*}, s\right) \in E_{0} \subseteq E$, hence $\left(t_{*}, s\right) \notin\left(A \times B_{i_{0}}\right) \backslash E=C$, that is $s \notin C_{t_{*}}$. Since for every $s \in C_{t_{*}} k\left(t_{*}, s\right)=0$ and for every $s \in\left(E_{0}\right)_{t_{*}} k\left(t_{*}, s\right) \geq \epsilon$, we have ess $\sup \left|k\left(t_{*}, s_{1}\right)-k\left(t_{*}, s_{2}\right)\right| \geq \epsilon$, which is the contradiction with /!!/. $s_{1}, s_{2} \in B_{i_{0}}$

Mention that the operator $T$ is neither $M$ - nor $L$-weakly compact. Moreover to note that by Dunford theorem ([1], p. 207) every bounded operator from $L_{1}$ into $L_{\infty}$ is integral.

By Lozanovsky theorem ([1], p. 199) for an arbitrary positive integral operator $T: X \rightarrow Y$, where $X$ and $Y$ are Banach function spaces associated with $\sigma$-finite measures, there exist a sequence of compact operators $K_{n}$ and a sequence of positive operators $S_{n}$ such that $0 \leq S_{n} \uparrow T$ and $S_{n} \leq K_{n}$. It is true, when only sequences goes over into nets, and for an arbitrary abstract integral operator $T: E \rightarrow F$, where $E$ and $F$ are Banach lattices, $F$ is Dedekind complete. By this reason, an Lozanovsky essential spectrum of a positive operator $T: E \rightarrow E$ is named the set

$$
\sigma_{\mathrm{el}}(T)=\bigcap_{\substack{0 \leq Q \leq T \\ Q \leq K \in \mathcal{K}(E)}} \sigma(T-Q) .
$$

Clearly $\sigma_{\text {el }}(T) \subseteq \sigma_{\text {ew }}^{-}(T)$. Under assumptions of theorem 5, it follows that the inclusion $r(T) \notin$ $\sigma_{\mathrm{ef}}(T)$ implies $r(T) \notin \sigma_{\mathrm{el}}(T)$. Turn out the opposite is true.

Theorem 7. Let $T$ be a positive operator on a Banach lattice $E$. If $r(T) \in \sigma_{\mathrm{ef}}(T)$ then $r(T) \in \sigma_{\text {el }}(T)$.

Proof. If $r(T)=0$ then $\sigma_{\mathrm{el}}(T)=\{0\}$, so $r(T) \in \sigma_{\mathrm{el}}(T)$. Thus we can suppose $r(T)=1$. Assume by the way of a contradiction, that is $1 \notin \sigma_{\mathrm{el}}(T)$. There exist a positive operator $Q$ and a compact operator $K$ such that $Q \leq T, Q \leq K$ and the operator $R=I-(T-Q)$ has the inverse. Then $r(T-Q)<1$ hence $R^{-1} \geq 0$, it follows that $0 \leq R^{-1} Q \leq R^{-1} K$. By 
Aliprantis-Burkinshaw theorem ([3], p. 275) the operator $\left(R^{-1} Q\right)^{3}$ is compact, so [7] $I-R^{-1} Q$ is a Fredholm operator. The relations $I-T=R\left(I-R^{-1} Q\right)$ and the index theorem ([1], p. 158 ) imply $I-T$ is a Fredholm operator, that is $1 \notin \sigma_{\text {ef }}(T)$, a contradiction.

It is not known if the inclusion $\sigma_{\mathrm{ef}}(T) \subseteq \sigma_{\mathrm{el}}(T)$ holds. Note that by Dodds-Fremlin theorem ([3], p. 279) if Banach lattices $E$ and $E^{*}$ have order continuous norms then $\sigma_{\mathrm{el}}(T)=\sigma_{\text {ew }}^{-}(T)$, therefore $\sigma_{\text {ef }}(T) \subseteq \sigma_{\text {el }}(T)$.

\section{Applications to the theory of band irreducible operators}

Band irreducible operators are one of most studied and important class of positive operators with the point of view of the spectral theory. As it should be results which are obtained above, are could be define more precisely for such operators. Moreover questions which are considered above, turns out closely connected with the next problem: when the inequalities $0 \leq S<T$ imply the spectral radius inequality $r(S)<r(T)$, where $T$ is band irreducible operator? It is true when a space is a finite dimensional. In the general case, the answer is negative even when $T$ is a band irreducible integral operator on any from spaces $\ell_{1}, \ell_{2}, \ell_{\infty}$ [2]. The next theorem completely closes this problem for the case of band irreducible abstract integral operators.

Theorem 8. Let $T$ be a band irreducible abstract integral operator on a Dedekind complete Banach lattice $E$, the dimension of $E$ is at least two and the $\sigma$-order continuous dual $E_{c}^{\sim} \neq\{0\}$. Then the next conditions are equivalent to each other:
(a) $r(T) \notin \sigma_{\mathrm{ef}}(T)$;
(b) $r(T) \notin \sigma_{\text {ew }}(T)$;
(c) $r(T) \notin \sigma_{\text {ew }}^{+}(T)$;
(d) $r(T) \notin \sigma_{\mathrm{el}}(T)$;
(e) the point $\lambda=r(T)$ is a pole of the resolvent $R(., T)$;
(f) for every operator $S$ the inequalities $0 \leq S<T$ imply $r(S)<r(T)$;

Moreover if T satisfies the condition (B), then (a) - (f) are equivalent to:

(g) $r(T) \notin \sigma_{\text {ew }}^{-}(T)$.

Proof. Implications (c) $\Rightarrow$ (b) $\Rightarrow(\mathrm{a}) \Rightarrow(\mathrm{e}),(\mathrm{f}) \stackrel{(\mathrm{B})}{\Rightarrow}(\mathrm{g}) \Rightarrow(\mathrm{b})$, (f) $\Rightarrow$ (d) are obvious, (d) $\Rightarrow$ (a) follows from theorem 7, (e) $\Rightarrow$ (f) is proven in [2]. The condition (e) implies [2] the condition (a) and the point $\lambda=r(T)$ is a simply pole of the resolvent $R(., T)$, by theorem 4 it implies (c).

In the more general case, when $T$ is a $\sigma$-order continuous band irreducible operator, the next theorem holds.

Theorem 8'. Let $E$ be a Banach lattice, the dimension of $E$ is at least two and the $\sigma$-order continuous dual $E_{c}^{\sim} \neq\{0\}$. Assume that $T$ is a band irreducible $\sigma$-order continuous operator on E. Then next implications are valid (see theorem 8):

$$
\text { (a) } \Longleftrightarrow(\mathrm{b}) \Longleftrightarrow(\mathrm{c}) \Longleftrightarrow(\mathrm{e}) \Longrightarrow \text { (f) } \stackrel{(\mathrm{B})}{\Longrightarrow}(\mathrm{g}) \Longrightarrow \text { (d) } \Longrightarrow \text { (a). }
$$

If $T$ is an arbitrary positive operator, implications (c) $\Rightarrow$ (b) $\Rightarrow$ (a) $\Rightarrow$ (e), (f) $\stackrel{(\mathrm{B})}{\Rightarrow}(\mathrm{g}) \Rightarrow$ (d) $\Rightarrow$ (a) and (g) $\Rightarrow$ (b) hold. Simply examples show that implications (a) $\Rightarrow$ (f), (e) $\Rightarrow$ (b) and (e) $\Rightarrow$ (d) don't hold. 


\section{References}

[1] Abramovich Y.A., Aliprantis C.D.: An invitation to the operator theory. Graduate studies in Mathematics. - Vol. 50. - 2002.

[2] Alekhno E.A.: Spectral properties of band irreducible operators. Proceedings Positivity IV - Theory and Application. Dresden (Germany). — 2006. — P. 5-14.

[3] Aliprantis C.D., Burkinshaw O.: Positive operators. Academic Press, 1985.

[4] Erovenko V.A.: Functional analysis: spectral and Fredholm properties of linear operators. (Russian) Minsk: BSU, 2002.

[5] Kato T.: Perturbation theory for linear operators. Springer-Verlag, 1976.

[6] Krasnoselskii M.A., Zabreiko P.P., Pustylnik E.I., Sobolevskii P.E.: Integral operators in spaces of summable functions. Noordhoff international publishing, Leyden, 1976.

[7] Latrach K., Jeribi A.: Some results on Fredholm operators, essential spectra and application. J. Math. Anal. and Appl. - 1998. - Vol. 225, No 2. — P. 461-485.

[8] Strizhevskii V.Z.: Disjointness of certain classes of order continuous operators. (Russian) Dokl. Akad. Nauk SSSR. - 1985. — Vol. 280, No 3. - P. 556-559. English translation: Soviet Math. Dokl. — 1985. - Vol. 31, No 1. - P. 128-130.

[9] Zaanen A.C.: Riesz spaces II. North-Holland: Amsterdam, 1983.

[10] Schaefer H.H.: Banach lattices and positive operators. Springer-Verlag, 1974.

[11] Erdös P., Oxtoby J.C.: Partitions of the plane into sets having positive measure in every non-null measurable product set. Trans. Amer. Math. Soc. — 1955. — Vol. 79, No 1. — P. 91-102.

Egor A. Alekhno

Belarussian State University

Faculty of Mechanics and Mathematics

Minsk, Belarus

E-mail: Alekhno@bsu.by 